\title{
Search for sterile neutrinos with the T2K far detector
}

\author{
Ka Ming Tsui ${ }^{* \dagger}$ \\ RCCN, ICRR, University of Tokyo \\ E-mail: kmtsui@icrr.u-tokyo.ac.jp
}

\begin{abstract}
T2K is a long baseline accelerator neutrino experiment in Japan which studies neutrino oscillations with a narrow-band muon neutrino beam peaked at $0.6 \mathrm{GeV}$. In the present study the T2K setup is used to search for light sterile neutrinos and constrain $\theta_{24}$ and $\theta_{34}$ mixing elements in the $3+1$ sterile neutrino model. This is the first long baseline study of sterile neutrinos at T2K which is based on SK data. To enhance the sensitivity to the effects related to the presence of sterile neutrinos, a joint analysis is done using both charged-current and the newly implemented neutral-current (NC) oscillation samples ( $\mathrm{NC} \pi^{0}$ with 2 rings observed and $\mathrm{NC}$ gamma de-excitation) at the far detector. The analysis strategy and the results obtained for the current T2K data (2010-2017 data taking) are presented. We set the most stringent limit on $\sin ^{2} \theta_{24}$ for the sterile neutrino mass splitting $\Delta m_{41}^{2}<3 \times 10^{-3} \mathrm{eV}^{2}$, and exclude $\sin ^{2} \theta_{24}>0.1$ and $\left|U_{\tau 4}\right|^{2}=\cos ^{2} \theta_{24} \sin ^{2} \theta_{34}>0.5$ at $90 \%$ C.L. for $\Delta m_{41}^{2}=0.1 \mathrm{eV}^{2}$.
\end{abstract}

The 39th International Conference on High Energy Physics (ICHEP2018)

4-11 July, 2018

Seoul, Korea

\footnotetext{
* Speaker.

${ }^{\dagger}$ On behalf of the $\mathrm{T} 2 \mathrm{~K}$ collaboration
} 


\section{Introduction}

A sterile neutrino is a neutral singlet fermion which can contribute to weak interactions only through mixing with active neutrinos. While their existence is still an open question, they are present in many extensions of the Standard Model and can have important implications on neutrino oscillation experiments, astrophysics, cosmology, etc. There are some hints of sterile neutrino coming from accelerator and reactor/radioactive source experiments suggesting an oscillation with a third mass splittings $\Delta m^{2} \sim 1 \mathrm{eV}^{2}$, which could be due to the existence of one or more sterile neutrinos. However, there are tensions between existing experimental results and no definitive conclusion has been reached.

While T2K is designed mainly for studying standard 3-flavor neutrino oscillation, specifically to look for oscillations at $\Delta m^{2} \sim 10^{-3} \mathrm{eV}^{2}$, it also has the potential to search for oscillation signatures due to sterile neutrinos. In this study, we use only the oscillation samples from the far detector Super-Kamiokande (SK) to perform the first long baseline search of sterile neutrino at T2K.

We consider the simplest $3+1$ model with only one sterile neutrino $v_{s}$ to constrain $\theta_{24}$ and $\theta_{34}$ mixing elements for a range of $\Delta m_{41}^{2}$. The $v_{\mu}$ survival probability and $v_{e}$ appearance probability at SK are modified by the sterile mixing as:

$$
\begin{gathered}
P\left(v_{\mu} \rightarrow v_{\mu}\right) \approx 1-\sin ^{2} 2 \theta_{23} \cos ^{4} \theta_{24} \sin ^{2} \frac{\Delta m_{31}^{2}}{4 E}-\sin ^{2} 2 \theta_{24} \sin ^{2} \frac{\Delta m_{41}^{2}}{4 E}, \\
P\left(v_{\mu} \rightarrow v_{e}\right) \approx \sin ^{2} 2 \theta_{13} \cos ^{2} \theta_{24} \sin ^{2} \theta_{23} \sin ^{2} \frac{\Delta m_{31}^{2}}{4 E},
\end{gathered}
$$

while the neutral-current (NC) channel can now exhibit oscillation behavior:

$$
P_{N C}=1-P\left(v_{\mu} \rightarrow v_{s}\right) \approx 1-\sin ^{2} \theta_{34} \sin ^{2} 2 \theta_{23} \sin ^{2} \frac{\Delta m_{31}^{2}}{4 E}-\cos ^{2} \theta_{34} \sin ^{2} 2 \theta_{24} \sin ^{2} \frac{\Delta m_{41}^{2}}{4 E} .
$$

\section{The T2K experiment}

The T2K (Tokai-to-Kamioka) experiment is a long baseline accelerator neutrino oscillation experiment in Japan. At the J-PARC facility in Tokai, a $30 \mathrm{GeV}$ proton beam is used to hit a graphite target, producing primarily $v_{\mu}$ in neutrino beam mode and $\bar{v}_{\mu}$ in antineutrino beam mode respectively. The beam is tuned in an off-axis angle of $2.5^{\circ}$ relative to the far detector such that the neutrino energy is narrowly peaked at $0.6 \mathrm{GeV}$ and has much less $v_{e} / \bar{v}_{e}$ contamination.

The neutrino beam is first measured by a set of near detectors at $280 \mathrm{~m}$ from the graphite target: INGRID is an array of iron/scintillator detectors on the center of the beam axis which measures precisely the beam direction and intensity; ND280 is the off-axis, magnetized tracking detector, which constrains the neutrino flux and cross-section model parameters in the oscillation analysis.

Our far detector SK at Kamioka detects the oscillated neutrino events at $295 \mathrm{~km}$ away. Apart from the charged-current (CC) oscillation samples used in the standard 3-flavor oscillation analysis [1], SK also provides a few NC samples that benefits the sterile search. They are the NC $\pi^{0}$ samples which select events with single $\pi^{0}$ production decaying through $\pi^{0} \rightarrow 2 \gamma$; and the NC gamma deexcitation sample where neutral-current quasi-elastic (NCQE) interaction between neutrino and oxygen produces ${ }^{15} \mathrm{~N}^{*} /{ }^{15} \mathrm{O}^{*}$, emitting de-excitation gammas peaked at $6 \mathrm{MeV}$ [2]. Deficit in these samples may be inferred as active neutrinos oscillating into sterile one. Fig. 1 shows the observed and expected event spectra in the absence of sterile mixing. 


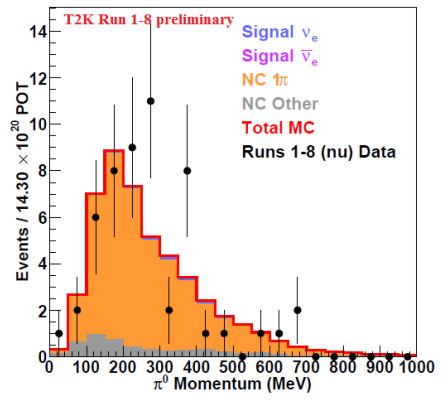

(a) $\mathrm{NC} \pi^{0}$, neutrino mode.

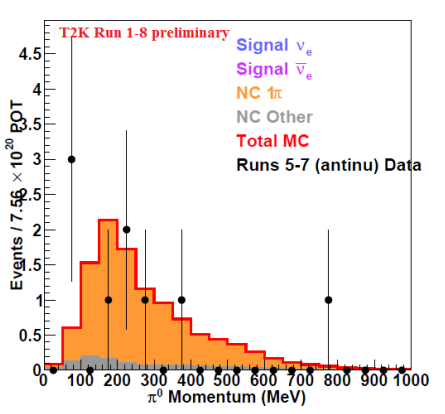

(b) $\mathrm{NC} \pi^{0}$, antineutrino mode.

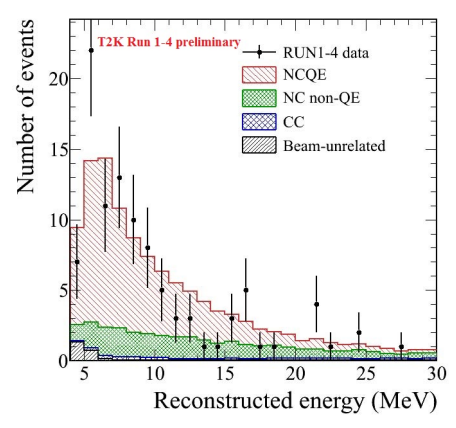

(c) NC gamma de-excitation, neutrino mode.

Figure 1: Event spectra of $\mathrm{NC} \pi^{0}$ samples (left and middle) and $\mathrm{NC}$ gamma de-excitation sample (right). MC prediction is calculated assuming no sterile mixing.

\section{Analysis method}

The analysis method used in this study is similar to the standard oscillation analysis in Ref.[1], except that we are fitting the sterile mixing parameters and gaining extra sensitivity from the new NC samples. The flux and cross-section measurements at ND280 (assuming no oscillation, which is valid for $\Delta m_{41}^{2}<0.3 \mathrm{eV}^{2}$ ) are used to predict the non-oscillated event spectra at SK, and the oscillation parameters are varied to achieve the best agreement with data. Additional cross-section (NC1 $\pi / \mathrm{NCQE}, \sim 30 \%)$ and far detector $(\sim 10 \%)$ errors are assigned to the NC samples, which are a bit larger than the $\mathrm{CC}$ samples.

\section{Results and prospects}

Table 1: Event rates of T2K Run 1-8 data and MC prediction assuming no sterile mixing.

\begin{tabular}{c|c|c|c|c|c|c|c|c}
\hline Sample & \multicolumn{2}{|c|}{ 1-Ring $\mu$} & \multicolumn{3}{|c|}{ 1-Ring $e$} & \multicolumn{2}{c|}{$\mathrm{NC} \pi^{0}$} & $\mathrm{NC} \gamma$ de-excit. \\
\hline Mode & $v$ & $\bar{v}$ & $v$ & $\bar{v}$ & $v 1 \pi^{+}$ & $v$ & $\bar{v}$ & $v$ \\
\hline Data & 240 & 68 & 74 & 7 & 15 & 53 & 9 & 102 \\
\hline Prediction & 268.4 & 64.3 & 73.5 & 7.9 & 6.9 & 49.5 & 11.3 & 107.7 \\
\hline
\end{tabular}

Data fits are performed in two parameter planes (see Fig. 2). With T2K Run 1-8 data, which corresponds to an exposure of $14.7(7.6) \times 10^{20}$ protons on target in neutrino (antineutrino) mode, we found no sterile neutrino and set the upper limits on the sterile mixing parameters. We obtained the best limit on $\sin ^{2} \theta_{24}$ for $\Delta m_{41}^{2}<3 \times 10^{-3} \mathrm{eV}^{2}$, with certain difference between normal hierarchy (NH, $m_{1}<m_{2}<m_{3}$ ) and inverted hierarchy ( $\mathrm{IH}, m_{3}<m_{1}<m_{2}$ ). But we are not competitive in the larger $\Delta m_{41}^{2}$ region because of the lack of high neutrino energy events. We exclude $\sin ^{2} \theta_{24}>0.1$ and $\left|U_{\tau 4}\right|^{2}=\cos ^{2} \theta_{24} \sin ^{2} \theta_{34}>0.5$ at $90 \%$ C.L. for $\Delta m_{41}^{2}=0.1 \mathrm{eV}^{2}$. Due to the limited statistics and large systematic errors of the NC samples, our limit on $\sin ^{2} \theta_{34}$ is worse than others.

Apart from future statistical updates, we are aiming to improve our analysis by better systematic studies on the NC samples. Additional multi-ring samples with higher neutrino energy and 

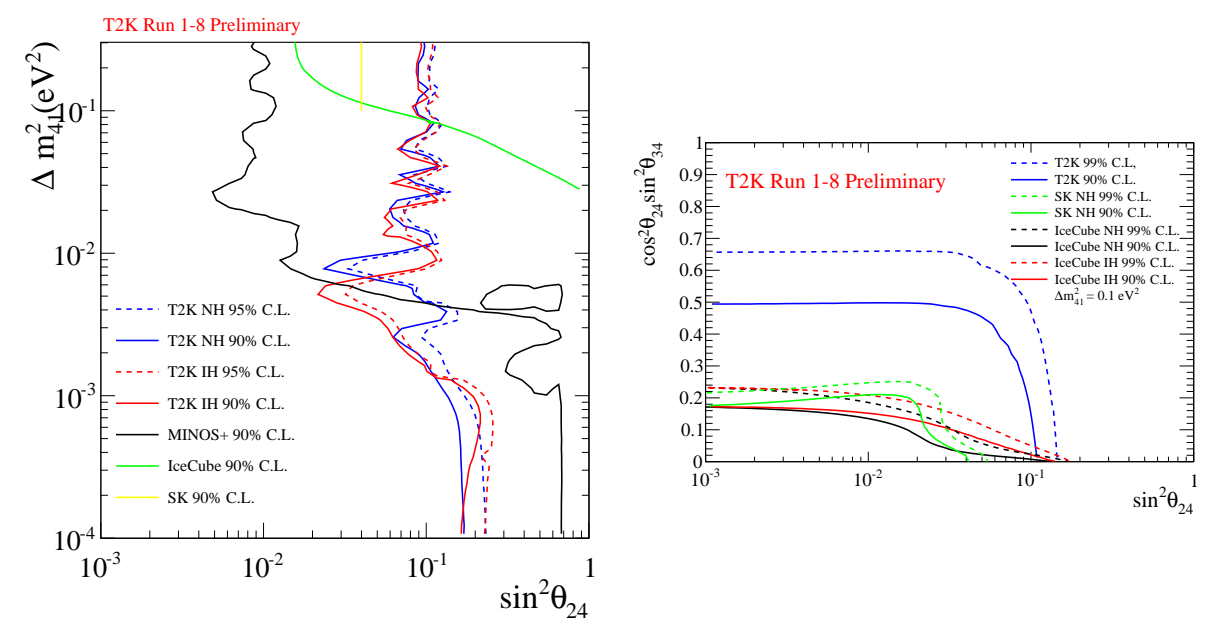

Figure 2: The T2K $90 \%$ exclusion limit on $\sin ^{2} \theta_{24}$ as a function of $\Delta m_{41}^{2}$ (left), and $90 \%$ and 99\% exclusion limit on $\sin ^{2} \theta_{24}$ vs. $\sin ^{2} \theta_{34}$ (right), together with results from other experiments $[3,4,5,6]$.

NC samples are in development to provide extra sensitivity in the interesting parameter space. A longer term planning is to combine this study with the ND280 analysis to perform a short + long baseline sterile neutrino search. Together with the coming upgrades for T2K-II, we will exploit our full discovery potential in both standard and sterile neutrino oscillation.

\section{References}

[1] T2K collaboration, K. Abe et al., Search for CP Violation in Neutrino and Antineutrino Oscillations by the T2K Experiment with $2.2 \times 10^{21}$ Protons on Target, Phys. Rev. Lett. 121 (2018) 171802 [1807.07891].

[2] T2K collaboration, K. Abe et al., Measurement of the neutrino-oxygen neutral-current interaction cross section by observing nuclear deexcitation $\gamma$ rays, Phys. Rev. D90 (2014) 072012 [1403. 3140 ].

[3] MINOS collaboration, P. Adamson et al., Search for sterile neutrinos in MINOS and MINOS+ using a two-detector fit, Submitted to: Phys. Rev. Lett. (2017) [1710.06488].

[4] SUPER-KAMIOKANDE collaboration, K. Abe et al., Limits on sterile neutrino mixing using atmospheric neutrinos in Super-Kamiokande, Phys. Rev. D91 (2015) 052019 [1410 . 2008 ].

[5] ICECUBE collaboration, M. G. Aartsen et al., Searches for Sterile Neutrinos with the IceCube Detector, Phys. Rev. Lett. 117 (2016) 071801 [1605.01990].

[6] ICECUBE collaboration, M. G. Aartsen et al., Search for sterile neutrino mixing using three years of IceCube DeepCore data, Phys. Rev. D95 (2017) 112002 [1702. 05160]. 